\title{
SÍNDROME DE TAKOTSUBO E O USO DO INTERTAK DIAGNOSTIC SCORE NO DIAGNÓSTICO DIFERENCIAL COM A SÍNDROME CORONARIANA AGUDA: RELATO DE UM CASO
}

\author{
TAKOTSUBO SYNDROME AND THE USE OF INTERTAK \\ DIAGNOSTIC SCORE IN THE DIFFERENTIAL DIAGNOSIS OF \\ ACUTE CORONARY SYNDROME: A CASE REPORT
}

\author{
Allana Maychat Pereira Oliveira ${ }^{1}$, Leonardo Silveira Nascimento ${ }^{1}$, \\ Paula Bibiana Müller Nunes ${ }^{1}$, Angélica Cristine Feil ${ }^{1}$, Júlia Cardoso Brum¹, \\ Simoni Lopes Peixoto², Nelson Ricardo Thomas Junior ${ }^{2}$
}

\section{RESUMO}

A Cardiomiopatia de Takotsubo (CT) é uma síndrome cardíaca caracterizada por quadro clínico compatível com síndrome coronariana aguda (SCA), com alterações eletrocardiográficas, aumento de enzimas cardíacas e anormalidades na contratilidade ventricular, geralmente associada a artérias coronárias livres de obstruções ou espasmos significativos à cineangiocoronariografia. Por apresentar curso clínico semelhante ao do infarto agudo do miocárdio, muitas vezes é abordada como tal. O presente trabalho busca, ao relatar um caso clássico de CT, destacar a importância da realização do diagnóstico diferencial entre a CT e a SCA, usando como ferramenta o InterTAK Diagnostic Score.

Palavras-chave: Cardiomiopatia de Takotsubo; cardiopatias; disfunção ventricular; estresse psicológico

\begin{abstract}
Takotsubo cardiomyopathy (TC) is a cardiac syndrome characterized by clinical symptoms compatible with acute coronary syndrome (ACS), including electrocardiographic changes, increased cardiac enzymes, and ventricular wall motion abnormalities, usually associated with absence of obstructive coronary artery disease or significant spasms at coronary angiography. Because its clinical course is similar to that of acute myocardial infarction, TC is often treated as such. The present paper reports a classic case of TC seeking to highlight the importance of performing a differential diagnosis between TC and ACS, using a tool named InterTAK Diagnostic Score.
\end{abstract}

Keywords: Takotsubo cardiomyopathy; heart diseases; ventricular dysfunction; stress; psychological

A Cardiomiopatia de Takotsubo (CT) é uma síndrome cardíaca caracterizada por sintomas torácicos, como dor e dispneia, e anormalidades reversíveis de contração da parede cardíaca do ventrículo esquerdo (VE) nos segmentos apical, médio, basal ou focal, o que confere a ele a aparência de um equipamento de pesca de polvo usado no Japão, chamado de Takotsubo, o qual determina o nome da síndrome ${ }^{1-3}$. Além disso, comumente cursa com anormalidades eletrocardiográficas e alteração dos marcadores bioquímicos de lesão miocárdica, tornando a síndrome um diagnóstico diferencial de síndrome coronariana aguda (SCA). Essa cardiomiopatia costuma ocorrer em mulheres na pós-menopausa e com frequência há histórico de estresse físico ou emocional precedendo o início dos sintomas ${ }^{1-5}$.

A European Society of Cardiology (ESC) recentemente publicou o Consenso Internacional sobre Síndrome de Takotsubo. Uma das atualizações foi quanto

Clin Biomed Res. 2018;38(4):409-413

1 Curso de Medicina, Universidade de Santa Cruz do Sul (UNISC). Santa Cruz do Sul, RS, Brasil.

2 Programa de Residência Médica em Clínica Médica, Hospital Santa Cruz. Santa Cruz do Sul, RS, Brasil.

Autor correspondente Allana Maychat Pereira Oliveira allanamaychat@gmail.com Hospital Santa Cruz Rua Fernando Abott, 174 96810-150, Santa Cruz do Sul, RS, Brasil. 
ao uso do InterTAK Diagnostic Score para estimar a probabilidade de um paciente apresentar CT e auxiliar no diagnóstico diferencial com SCA com alta sensibilidade e especificidade. Esse escore consiste em sete variáveis clínicas e eletrocardiográficas (Tabela 1) e estratifica os pacientes em baixa ( $\leq 70$ pontos) e alta probabilidade ( $>70$ pontos) de apresentarem CT. Aqueles com baixo risco devem ser encaminhados para a cineangiocoronariografia, enquanto que aqueles com alto risco devem realizar ecocardiografia transtorácica ${ }^{3,4}$.

Apenas cerca de $2 \%$ dos pacientes com suspeita de SCA recebem como diagnóstico final a $\mathrm{CT}^{7}$. O objetivo desse trabalho é descrever um caso de Síndrome de Takotsubo, ressaltando a importância de incluí-la como diagnóstico diferencial nas suspeitas de SCA, utilizando como ferramenta o InterTAK Diagnostic Score.

\section{APRESENTAÇÃO DO CASO}

Paciente do sexo feminino, parda, 58 anos, agricultora, previamente hipertensa em tratamento medicamentoso, procurou atendimento de urgência por quadro de dor precordial em aperto, de forte intensidade, ventilatório-dependente, com irradiação para membro superior esquerdo, após estresse emocional agudo no dia anterior (perda total de sua colheita após incêndio criminoso no local).

À admissão encontrava-se em bom estado geral, visivelmente ansiosa, com pressão arterial de 200/120 mmHg, frequência de cardíaca de 116 bpm, frequência respiratória de 24 irpm, ausculta cardíaca com ritmo regular, em dois tempos, com bulhas normofonéticas e sem sopros audíveis, ausculta pulmonar com murmúrios vesiculares uniformemente distribuídos, sem ruídos adventícios e sem outras alterações ao exame físico.

Iniciada investigação através da realização de um eletrocardiograma, que revelou ritmo sinusal e bloqueio de ramo esquerdo, e da dosagem dos marcadores de necrose miocárdica. A Tabela 2 apresenta a evolução dos marcadores de lesão miocárdica durante a internação. Ao InterTAK Diagnostic Score, a paciente somou 61 pontos (sexo feminino, estresse emocional e ausência de infradesnivelamento do segmento ST).

Instituído manejo para SCA, com ácido acetilsalicílico, clopidogrel, heparina de baixo peso molecular, succinato de metoprolol, atorvastatina, enalapril e mononitrato de isossorbida. Em seguida, a paciente foi encaminhada para o centro de alta complexidade de referência, onde realizou cineangiocoronariografia, a qual evidenciou dominância coronariana direita e coronárias epicárdicas livres de lesões obstrutivas significativas (Figura 1) e a ventriculografia revelou hipocinesia em região apical, com disfunção sugestiva de baloneamento apical (Figura 2).

A paciente apresentou boa evolução clínica durante a hospitalização, recebendo alta no segundo dia de internação, com melhora completa da dor torácica e com normalização dos marcadores de necrose miocárdica. Ecocardiograma transtorácico realizado três meses após a alta mostrou função sistólica do VE completamente restaurada.

Tabela 1: InterTAK Diagnostic Score.

\begin{tabular}{lc}
\hline \multicolumn{1}{c}{ Variáveis } & Pontos \\
\hline Sexo feminino & 25 \\
Estresse emocional & 24 \\
Estresse físico & 13 \\
Ausência de infradesnivelamento do segmento ST & 12 \\
Doença psiquiátrica pregressa, aguda ou crônica & 11 \\
Doença neurológica pregressa, aguda ou crônica & 9 \\
Prolongamento do intervalo QT (mulheres > 460ms; homens > 440ms) & 6 \\
\hline A poça & 9 \\
\hline
\end{tabular}

A pontuação varia de 0 a 100 pontos. Um escore acima de 70 pontos estratifica o paciente como de alta probabilidade para Síndrome de Takotsubo. O InterTAK Diagnostic Score está disponível para acesso online ${ }^{6}$.

Tabela 2: Evolução dos biomarcadores de lesão miocárdica conforme o tempo decorrido desde a admissão hospitalar.

\begin{tabular}{lcccc}
\hline \multirow{2}{*}{ Marcadores } & \multicolumn{3}{c}{ Tempo da admissão } & \multirow{2}{*}{ VR $^{*}$} \\
\cline { 2 - 4 } & $\mathbf{0 h}$ & $\mathbf{6 h}$ & $\mathbf{1 2 h}$ & $30-145$ \\
CK total $(\mathrm{U} / \mathrm{l})$ & 151 & 132 & 78 & $<24$ \\
CK-MB $(\mathrm{U} / \mathrm{l})$ & 28 & 16 & 10 & $\leq 0,04$ \\
Troponina I $(\mathrm{ng} / \mathrm{ml})$ & 0,74 & 0,56 & 0,30 & \\
\hline
\end{tabular}

*Valor de referência. 

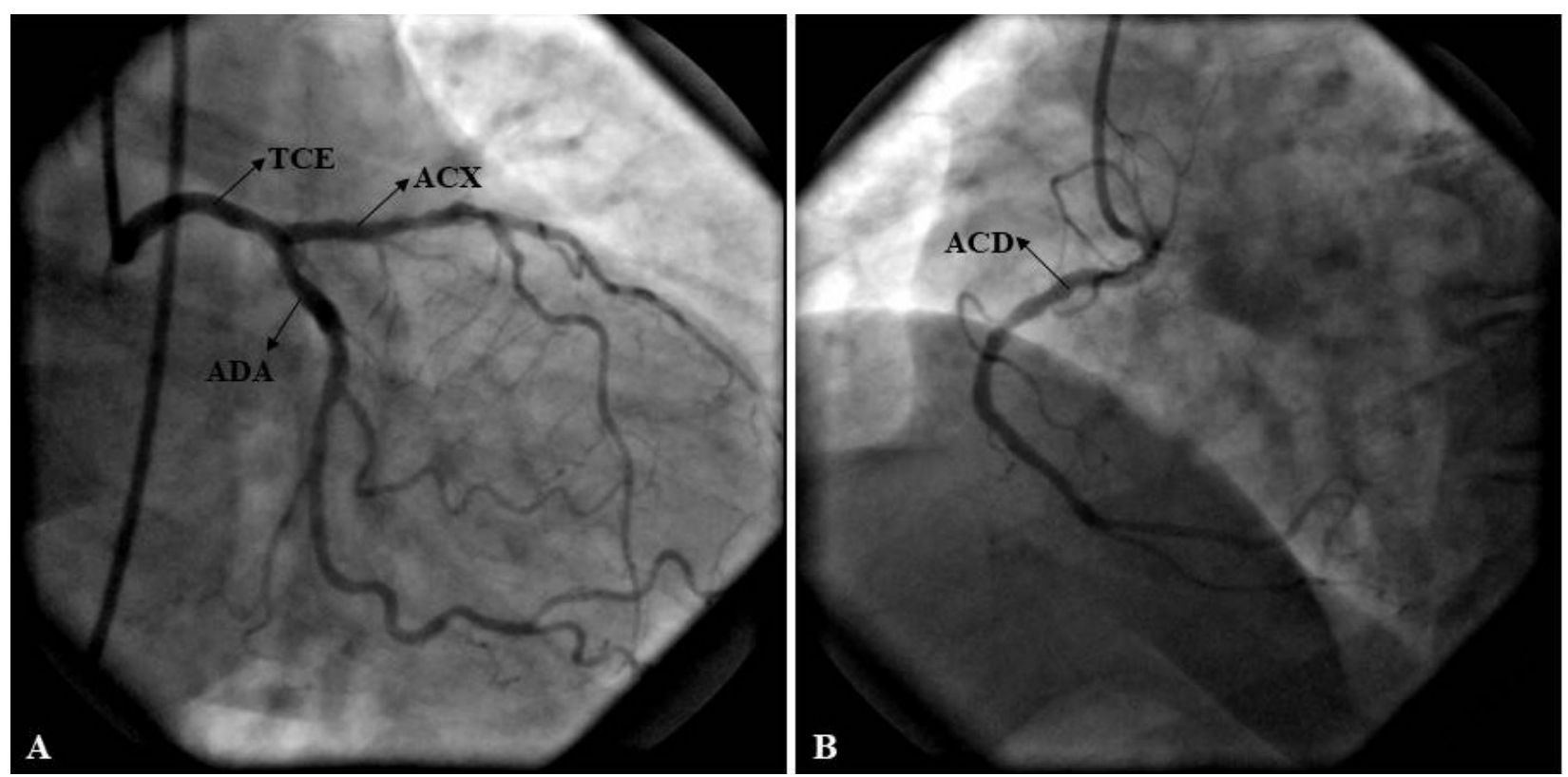

Figura 1: Cineangiocoronariografia esquerda $(A)$ e direita $(B)$ mostrando perviedade das artérias coronárias epicárdicas. $\mathrm{TCE}$ = tronco da coronária esquerda; $\mathrm{ACD}$ = artéria coronária direita; $\mathrm{ACX}$ = artéria circunflexa; $\mathrm{ADA}=$ artéria descendente anterior.

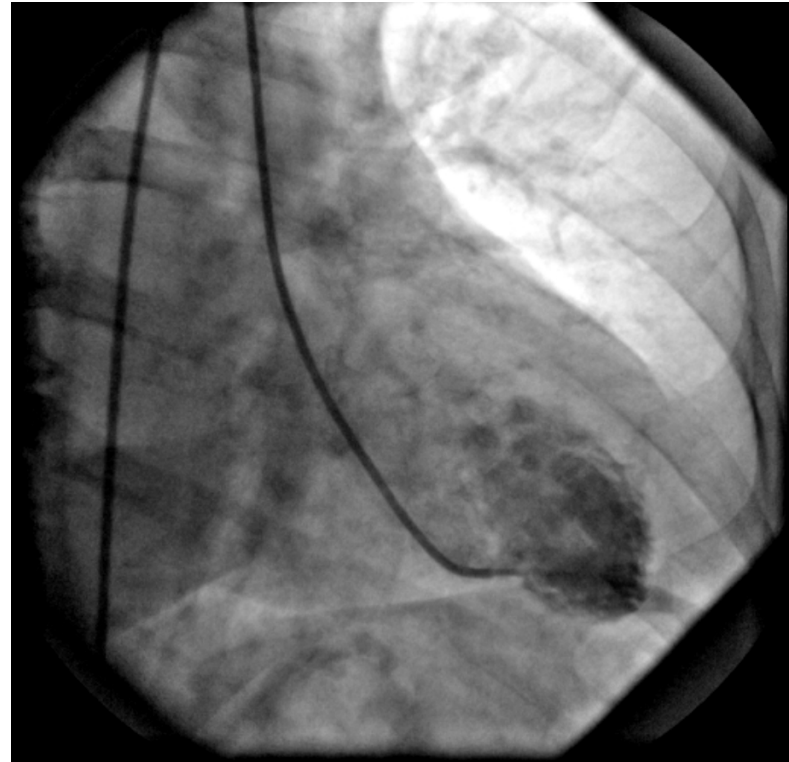

Figura 2: Ventriculografia mostrando abaulamento apical do ventrículo esquerdo durante a diástole máxima.

\section{DISCUSSÃO}

Também conhecida como Cardiomiopatia Induzida por Estresse, Síndrome do Coração Partido, Síndrome de Discinesia Apical Transitória e Síndrome do Baloneamento Apical ${ }^{2,8-10}$, a CT foi descrita originalmente no Japão no início da década de 1990 e tem sido cada vez mais reconhecida e diagnosticada nas diversas etnias ${ }^{2,7,10,11}$. Em diversos estudos a predominância das pessoas acometidas é do sexo feminino e a faixa etária afetada com maior frequência é a dos 62 a 76 anos $^{2,4,5,7}$. Com relação a fisiopatologia da doença, ainda não se sabe a etiologia, mas a teoria mais aceita atualmente é a de que há um excesso de catecolaminas ${ }^{3,8,10,12,13}$

A CT pode se apresentar em sua forma típica, com baloneamento ventricular apical, ou através de formas atípicas, que incluem o acometimento focal, basal ou médio-lateral do ventrículo. O reconhecimento destes padrões é essencial, tendo em vista que nas formas atípicas de CT há, com maior frequência, a presença de doenças neurológicas, menores níveis de peptídeo natriurético cerebral, maior probabilidade de haver infradesnivelamento do segmento ST, menor impacto na fração de ejeção do VE e tendem a ser observadas mais frequentemente em pacientes mais jovens. Além disso, os pacientes com a forma típica tem maior mortalidade no primeiro ano. Após esse prazo os valores de mortalidade se igualam entre os padrões típico e atípicos de $\mathrm{CT}^{3}$.

AESC, no seu último Consenso Internacional sobre Síndrome de Takotsubo, trouxe uma atualização nos critérios diagnósticos da CT. Estes incluem disfunção transitória do VE com hipocinesia, discinesia ou acinesia, podendo ou não haver envolvimento do ventrículo direito; história de estresse físico ou emocional, fator que não é obrigatório; anormalidades eletrocardiográficas; elevação dos biomarcadores de lesão miocárdica; e exclusão de miocardite infecciosa ${ }^{3,4}$. Diferentemente dos antigos critérios 
diagnósticos, essa atualização destaca que a CT pode coexistir com doença arterial coronariana e pode ainda ser desencadeada por comorbidades neurológicas e feocromocitoma ${ }^{3,7,11}$.

Com relação ao tratamento da $\mathrm{CT}$, não há um tratamento padrão recomendado e não deve ser realizada trombólise. Atualmente se preconiza a instituição imediata da terapêutica farmacológica preconizada para os casos de infarto agudo do miocárdio (dupla antiagregação plaquetária, nitratos, heparina e betabloqueadores) e, após o diagnóstico de CT ter sido estabelecido, devem ser suspensos os nitratos e antiagregação plaquetária e iniciado o uso de um inibidor da enzima conversora da angiotensina ${ }^{1,4,7,8,12}$. Nos casos em que há falência circulatória não se deve usar beta-agonistas ou vasopressores, sendo a terapêutica preferencial para esses casos o suporte circulatório mecânico ou ainda levosimendan ${ }^{4,12,13}$.

A CT originalmente era vista como benigna, mas os últimos estudos têm demonstrado que há uma mortalidade compatível com a da SCA, assim como há uma taxa de progressão para choque cardiogênico similar entre esses eventos ${ }^{3,4,14-16}$. Porém, a taxa de recorrência da CT é de aproximadamente $5 \%$ e tende a ocorrer no terceiro mês após o primeiro evento $^{4}$. De maneira geral, a função ventricular se recupera de forma integral, assim como há o desaparecimento dos sintomas, das alterações eletrocardiográficas e dos biomarcadores em um período de 6 a 8 semanas 8 ,10-13.

O caso apresentado foi um caso clássico de CT. A realização de cineangiocoronariografia foi adequada, tendo em vista que a paciente apresentou baixa probabilidade para CT ao InterTAK Diagnostic Score. O tratamento instituído esteve de acordo com o recomendado e a evolução rápida e favorável da paciente seguiu o estabelecido na literatura. Dessa forma, se ressalta a importância de incluir a CT como diagnóstico diferencial de dor torácica aguda, com o intuito de evitar a realização de trombólises desnecessárias e atentar ao manejo dos pacientes com instabilidade hemodinâmica decorrente da Síndrome de Takotsubo.

\section{Conflitos de Interesse}

Os autores declaram não haver conflito de interesse.

\section{REFERÊNCIAS}

1. Kurisu S, Kihara Y. Clinical management of takotsubo cardiomyopathy. Circ J. 2014;78(7):1559-66. http://dx.doi. org/10.1253/circj.CJ-14-0382. PMid:24964980.

2. Veillet-Chowdhury M, Hassan SF, Stergiopoulos K. Takotsubo cardiomyopathy: a review. Acute Card Care. 2014;16(1):15-22. http://dx.doi. org/10.3109/17482941.2013.869346. PMid:24552225.

3. Ghadri JR, Wittstein IS, Prasad A, Sharkey S, Dote K, Akashi YJ, et al. International Expert Consensus Document on Takotsubo Syndrome (Part I): clinical characteristics, diagnostic criteria and pathophysiology. Eur Heart $J$. 2018;39(22):2032-46. http://dx.doi. org/10.1093/eurheartj/ehy076. PMid:29850871.

4. Ghadri JR, Wittstein IS, Prasad A, Sharkey S, Dote K, Akashi YJ, et al. International Expert Consensus Document on Takotsubo Syndrome (Part II): diagnostic workup, outcome and management. Eur Heart J. 2018;39(22):2047-62. http://dx.doi. org/10.1093/eurheartj/ehy077. PMid:29850820.
5. Dahlviken RM, Fridlund B, Mathisen L. Women's experiences of Takotsubo cardiomyopathy in a short-term perspective - a qualitative content analysis. Scand J Caring Sci. 2015;29(2):258-67. http:// dx.doi.org/10.1111/scs. 12158 . PMid:24953349.

6. TakoTsubo International Registry. InterTAK Diagnostic Score. 2011 [citado 2018 Set 03]. Disponível em: www.takotsubo-registry.com/ takotsubo-score.html

7. Scantlebury DC, Prasad A. Diagnosis of Takotsubo cardiomyopathy. Circ J. 2014;78(9):2129-39. http://dx.doi. org/10.1253/circj.CJ-14-0859. PMid:25131525.

8. Reis JGV, Rosas G. Cardiomiopatia de Takotsubo: um diagnóstico diferencial da síndrome coronariana aguda: revisão de literatura. Rev Med Minas Gerais. 2010;20(4):594600 .

9. Merchant EE, Johnson SW, Nguyen P, Kang C, Mallon WK. takotsubo cardiomyopathy: a case series and review of the literature. WestJEM. 2008;9(2):104-11. PMid:19561716.
10. Rihl MF, Haas P, Holthausen RS. Cardiomiopatia de Takotsubo: relato de caso. Rev AMRIGS. 2015;59(1):30-4.

11. Kawai S, Kitabatake A, Tomoike H. Cardiomyopathy Group. Guidelines for diagnosis of takotsubo (ampulla) cardiomyopathy. Circ J. 2007;71(6):990-2. http:// dx.doi.org/10.1253/circj.71.990. PMid:17527002.

12. Nóbrega S, Brito D. Miocardiopatia Takotsubo: estado da arte. Rev Port Cardiol. 2012;31(9):589-96. http:// dx.doi.org/10.1016/j.repc.2012.02.014. PMid:22795894.

13. Silva VLLG, Hisano DK, Aquino RTR, Magliari MER, Almeida JAF, Almeida JD, et al. Broken Heart Syndrome (Síndrome de Takotsubo, Cardiomiopatia do estresse): relato de caso. Arq Med Hosp Fac Cienc Med Santa Casa São Paulo. 2008;53(3):125-9.

14. Tornvall P, Collste O, Ehrenborg $E$, Järnbert-Petterson H. A CaseControl Study of Risk Markers and Mortality in Takotsubo Stress 
Cardiomyopathy. J Am Coll Cardiol. 2016;67(16):1931-6. http://dx.doi. org/10.1016/j.jacc.2016.02.029.

PMid:27102508.

15. Stiermaier T, Eitel C, Desch S, Fuernau G, Schuler G, Thiele

$\mathrm{H}$, et al. Incidence, determinants and prognostic relevance of cardiogenic shock in patients with Takotsubo cardiomyopathy. Eur Heart J Acute Cardiovasc Care. 2016;5(6):489-96. http://dx.doi. org/10.1177/2048872615612456. PMid:26474843.
16. Templin C, Ghadri JR, Diekmann J, Napp LC, Bataiosu DR, Jaguszewski $\mathrm{M}$, et al. Clinical Features and Outcomes of Takotsubo (Stress) Cardiomyopathy. N Engl J Med. 2015;373(10):929-38. http://dx.doi. org/10.1056/NEJMoa1406761. PMid:26332547.

Recebido: 3 set, 2018 Aceito: 22 jan, 2019 